\title{
GENERALIZED LERAY-SCHAUDER PRINCIPLES FOR COMPACT ADMISSIBLE MULTIFUNCTIONS
}

\author{
SEHIE PARK \\ Dedicated to Professor Ky Fan on his 80th birthday
}

\section{Introduction}

We establish the Leray-Schauder type theorems for very general classes of multifunctions, which are called admissible. Our admissible classes contain compositions of important multifunctions in nonlinear analysis and algebraic topology. Moreover, our arguments are elementary, without using the concept of degree of maps or theory of homotopy extensions.

The Leray-Schauder principle [LS], one of the most important theorems in nonlinear analysis, was first proved for a Banach space in the context of degree theory. In $[\mathrm{N}]$, Nagumo extended the degree theory to locally convex topological vector spaces and his results can be used to generalize the Leray-Schauder principle. Variations of the principle were due to Browder $[\mathrm{B}]$ for Banach spaces and to Schaefer [Sc2] for locally convex topological vector spaces without using degree theory. Schaefer's version has important applications to integral equations. Later Potter $[\mathrm{Po}]$ generalized the results of Browder and Schaefer. However, those authors considered single-valued maps and adopted boundary conditions particular to the so-called Leray-Schauder condition (LS). There are many other authors who obtained generalized versions of the Leray-Schauder type theorems (see the references).

1991 Mathematics Subject Classification. 47H10, 54C60, 54H25, 55M20.

Supported in part by KOSEF-951-0102-006-2.

(C)1995 Juliusz Schauder Center for Nonlinear Studies 
Motivated by the works of Potter [Po] and Fitzpatrick and Petryshyn [FP], we prove two Leray-Schauder type theorems for admissible classes of compact multifunctions with (LS) without using the degree theory. Our results generalize many of well-known theorems of this type.

\section{Preliminaries}

A multifunction or map $F: X \rightarrow 2^{Y}$ is a function from a set $X$ into the set $2^{Y}$ of nonempty subsets of $Y$; that is, a function with values $F x \subset Y$ for $x \in X$ and fibers $F^{-} y=\{x \in X: y \in F x\}$ for $y \in Y$. For $A \subset X$, let $F(A)=\bigcup\{F x: x \in A\}$. A map $F: X \rightarrow 2^{Y}$ is compact provided $F(X)$ is contained in a compact subset of $Y$. For any $B \subset Y$, the (lower) inverse of $B$ under $F$ is defined by

$$
F^{-}(B)=\{x \in X: F x \cap B \neq \emptyset\} .
$$

Given two maps $F: X \rightarrow 2^{Y}$ and $G: Y \rightarrow 2^{Z}$, the composition $G F: X \rightarrow 2^{Z}$ is defined by $(G F) x=G(F x)$ for $x \in X$.

For topological spaces $X$ and $Y$, a map $F: X \rightarrow 2^{Y}$ is upper semicontinuous (u.s.c.) if, for each closed set $B \subset Y, F^{-}(B)$ is closed in $X$.

Note that compositions of u.s.c. maps are u.s.c. and that the image of a compact set under an u.s.c. map with compact values is compact.

Let - Int, and $\mathrm{Bd}$ denote the closure, interior, and boundary, resp.

In the sequel, a t.v.s. means a Hausdorff topological vector space. In a t.v.s., a convex hull of any finite subset will be called a polytope.

Given a class $\mathbb{X}$ of maps, $\mathbb{X}(X, Y)$ denotes the set of maps $F: X \rightarrow 2^{Y}$ belonging to $\mathbb{X}$, and $\mathbb{X}_{\mathrm{c}}$ the set of finite compositions of maps in $\mathbb{X}$.

A class $\mathfrak{A}$ of maps is one satisfying the following properties:

(i) $\mathfrak{A}$ contains the class $\mathbb{C}$ of (single-valued) continuous functions;

(ii) each $F \in \mathfrak{A}_{\mathrm{c}}$ is u.s.c. and compact-valued; and

(iii) for any polytope $P$, each $F \in \mathfrak{A}_{\mathrm{c}}(P, P)$ has a fixed point.

Examples of $\mathfrak{A}$ are $\mathbb{C}$, the Kakutani maps $\mathbb{K}$ (with convex values), the Aronszajn maps $\mathbb{M}$ (with $R_{\delta}$ values), the acyclic maps $\mathbb{V}$ (with acyclic values), the O'Neill maps $\mathbb{N}$ (with values consisting of one or $m$ acyclic components, where $m$ is fixed), the approachable maps $\mathbb{A}$ in a t.v.s., admissible maps in the sense of Górniewicz, permissible maps of Dzedzej, and others. For details, see [Gr3] and $[\mathrm{PK}]$.

We introduce two more classes:

$F \in \mathfrak{A}_{\mathrm{c}}^{\sigma}(X, Y) \Leftrightarrow$ for any $\sigma$-compact subset $K$ of $X$, there is an $\widetilde{F} \in \mathfrak{A}_{\mathrm{c}}(K, Y)$ such that $\widetilde{F} x \subset F x$ for each $x \in K$.

$F \in \mathfrak{A}_{\mathrm{c}}^{\kappa}(X, Y) \Leftrightarrow$ for any compact subset $K$ of $X$, there is an $\widetilde{F} \in \mathfrak{A}_{\mathrm{c}}(K, Y)$ such that $\widetilde{F} x \subset F x$ for each $x \in K$. 
Note that $\mathfrak{A} \subset \mathfrak{A}_{\mathrm{c}} \subset \mathfrak{A}_{\mathrm{c}}^{\sigma} \subset \mathfrak{A}_{\mathrm{c}}^{\kappa}$. Any class $\mathfrak{A}_{\mathrm{c}}^{\kappa}$ will be called admissible. For details and examples, see $[\mathrm{P} 1, \mathrm{PK}]$.

The following recent theorem is due to the author [P2, Theorem 4]:

TheOREM 1. Let $X$ be a nonempty convex subset of a locally convex t.v.s. $E$, and $F \in \mathfrak{A}_{\mathrm{c}}^{\sigma}(X, X)$. If $F$ is compact, then $F$ has a fixed point.

\section{Main results}

In this section, we prove two Leray-Schauder type theorems for compact admissible maps.

The following is a fixed point theorem for compact admissible maps satisfying the Leray-Schauder boundary condition:

TheOREM 2. Let $E$ be a locally convex t.v.s., $U$ a convex neighborhood of the origin 0 of $E$, and $X$ a convex subset of $E$ containing 0 . Then any compact map $F \in \mathfrak{A}_{\mathrm{c}}^{\sigma}(\bar{U} \cap X, X)$ satisfying

(LS) $F x \cap\{\lambda x: \lambda>1\}=\emptyset$ for each $x \in \operatorname{Bd}_{X} U$

has a fixed point.

Proof. Let $p$ be the Minkowski functional of $U$. Since $0 \in \operatorname{Int} U, p$ is continuous. Define $r: E \rightarrow \bar{U}$ by $r(x)=x$ for $x \in \bar{U}$ and $r(x)=p(x)^{-1} x$ for $x \notin \bar{U}$; that is,

$$
r(x)=[\max \{1, p(x)\}]^{-1} x \quad \text { for } x \in E .
$$

Then $r$ is a continuous retraction of $E$ onto $\bar{U}$. Moreover, since $X$ is convex and $0 \in X$, we have $r(X) \subset X$ and $r(F(\bar{U} \cap X)) \subset \bar{U} \cap X$. Define $G=r F$. Then $G \in \mathfrak{A}_{\mathrm{c}}^{\sigma}(\bar{U} \cap X, \bar{U} \cap X)$ and is compact. Therefore, by Theorem 1 , there exists an $x \in \bar{U} \cap X$ such that $x \in G x$; that is, $x=r(y)$ for some $y \in F x$. There are two possibilities: (i) $x \in \operatorname{Int} U$ or (ii) $x \in \operatorname{Bd}_{X} U$.

Suppose (i) holds. Then

$$
1>p(x)=p(r(y))=[\max \{1, p(y)\}]^{-1} p(y)
$$

Hence $p(y)<1$ and this implies $r(y)=y$. Then $x=r(y)=y \in F x$.

Suppose (ii) holds. Then

$$
1=p(x)=p(r(y))=[\max \{1, p(y)\}]^{-1} p(y)
$$

If $p(y)<1$, we have a contradiction. If $p(y)>1$, then $x=r(y)=p(y)^{-1} y$ and $y=p(y) x$. This contradicts (LS). Thus $p(y)=1$, so that $r(y)=y$ and $x=r(y)=y \in F x$. This completes our proof. 
Particular forms. 1. For the case $X=E$, Theorem 2 extends earlier results of Schaefer [Sc2], Granas [Gr1], Yamamuro [Y], Kaniel [Kn], Powers [Pw], Potter [Po], Ma [M], Martelli [Mr], Furi-Martelli [FM], Reich [R], and Park [P2] for particular classes in $\mathfrak{A}_{\mathrm{c}}^{\sigma}$.

2. For the case of $X$ not necessarily equal to $E$, Theorem 2 also includes earlier results of Fitzpatrick-Petryshyn [FP], Hahn [H], Granas [Gr2], GórniewiczGranas-Kryszewski [GGK], and Kaczyński-Wu [KW].

3. Recently, Granas [Gr3, Theorems 2.2 and 2.3] obtained Theorem 2 for normed vector spaces for the case $X=E$. He also gave possible generalizations of Theorem 2 to coincidence theorems and others.

The following type of theorems are usually known as the Leray-Schauder principle:

Theorem 3. Let $E$ be a locally convex t.v.s., $U$ a convex neighborhood of the origin 0 of $E$, and $X$ a cone of $E$. Let $H \in \mathfrak{A}_{\mathrm{c}}([0,1] \times(\bar{U} \cap X), X)$ be a compact map such that

(1) $x \notin H(t, x)$ for $t \in[0,1)$ and $x \in \operatorname{Bd}_{X} U$; and

(2) $H(0, x) \cap\{\lambda x: \lambda>1\}=\emptyset$ for $x \in \operatorname{Bd}_{X} U$.

Then there is a $z \in \bar{U} \cap X$ such that $z \in H(1, z)$.

Proof. Consider the maps $H_{n}: \bar{U} \cap X \rightarrow 2^{X}, n=1,2, \ldots$, defined by

$$
H_{n} x= \begin{cases}H\left((1-p(x)) / \varepsilon_{n}, x / p(x)\right), & 1-\varepsilon_{n} \leq p(x) \leq 1, \\ H\left(1, x /\left(1-\varepsilon_{n}\right)\right), & p(x)<1-\varepsilon_{n},\end{cases}
$$

where $p$ is the Minkowski functional of $U$ and $\left\{\varepsilon_{n}\right\}$ is a sequence of real numbers such that $\varepsilon_{n} \rightarrow 0$ as $n \rightarrow \infty$ and $0<\varepsilon_{n}<1 / 2$ for all $n$.

For each $n$, it is clear that $H_{n} \in \mathfrak{A}_{\mathrm{c}}(\bar{U} \cap X, X)$ and is compact. Also note that (2) implies

(LS) $H_{n} x \cap\{\lambda x: \lambda>1\}=\emptyset$ for each $x \in \operatorname{Bd}_{X} U$.

Now by Theorem 2 , for each $n$, there exists a $y_{n} \in \bar{U} \cap X$ such that $y_{n} \in H_{n} y_{n}$. Note that all $y_{n}$ belong to the range of $H$, which is relatively compact. Let $z$ be a cluster point of $\left\{y_{n}\right\}$. Since $\bar{U} \cap X$ is closed, we have $z \in \bar{U} \cap X$. Suppose that infinitely many $y_{n}$ satisfy

$$
1 \geq p\left(y_{n}\right) \geq 1-\varepsilon_{n}
$$

Without loss of generality, we may suppose that this is true for all $n$, and that

$$
t_{n}=\left(1-p\left(y_{n}\right)\right) / \varepsilon_{n} \rightarrow t \in[0,1] \quad \text { as } n \rightarrow \infty .
$$

Since $p\left(y_{n}\right) \rightarrow 1$, the point $(t, z, z)$ is a cluster point of the sequence $\left\{\left(t_{n}, y_{n} / p\left(y_{n}\right)\right.\right.$, $\left.\left.y_{n}\right)\right\}$ in $[0,1] \times(\bar{U} \cap X) \times(\bar{U} \cap X)$. Since $H$ is u.s.c. and compact, it has the 
closed graph. Hence, $y_{n} \in H\left(t_{n}, y_{n} / p\left(y_{n}\right)\right)$ implies $z \in H(t, z)$. This contradicts (1) since $p(z)=\lim p\left(y_{n}\right)=1$, so that $z \in \mathrm{Bd}_{X} \bar{U}$.

Thus $(*)$ is false and we may assume that $p\left(y_{n}\right)<1-\varepsilon_{n}$ for all $n$. Since $1-\varepsilon_{n} \rightarrow 1$ as $n \rightarrow \infty, y_{n} \in H\left(1, y_{n} /\left(1-\varepsilon_{n}\right)\right)$ implies $z \in H(1, z)$ by the closedness of the graph of $H$. This completes our proof.

Particular forms. For $X=E$, we have the following particular forms of Theorem 3.

1. The origin of Theorem 3 is essentially due to Leray and Schauder [LS] for a Banach space $E$ and $f \in \mathbb{C}$ in the context of degree theory.

2. Browder [B, Lemma 24] obtained a very particular form of Theorem 3 for a Banach space $E$ and $H=f \in \mathbb{C}$ with the boundary condition

$(2)^{\prime} f(0, x)=0$ for all $x \in \bar{U}$.

3. Potter [Po, Theorem] extended Browder's result for the boundary condition

$(2)^{\prime \prime} f(\{0\} \times \operatorname{Bd} U) \subset \bar{U}$.

From Theorem 3, we obtain the following:

Corollary 3.1. Let $E$ be a locally convex t.v.s. and $F \in \mathfrak{A}_{\mathrm{c}}(E, E)$ a compact map such that

(*) $\quad H \in \mathfrak{A}_{\mathrm{c}}([0,1] \times E, E)$, where $H(t, x)=t F x$ for $t \in[0,1]$ and $x \in E$.

Then either, for each $\lambda \in(0,1)$, there exists an $x \in E$ such that $x \in \lambda F x$, or the set $A:=\{x \in E: x \in \mu F x$ for some $\mu \in(0,1)\}$ is not bounded.

Proof. Let $\lambda \in(0,1)$ and $G:=\lambda F$ be defined by $G x=\lambda F x$ for $x \in E$. Suppose that $A$ is bounded. Then for any open convex neighborhood $V$ of 0 , there exists an $r>0$ such that $U:=r V \supset A$. Define $H \in \mathfrak{A}_{\mathrm{c}}([0,1] \times \bar{U}, E)$ by

$$
H(t, x)=t G x \quad \text { for } t \in[0,1] \text { and } x \in \bar{U} .
$$

Then $H$ is compact. Now we apply Theorem 3 with $X=E$. We claim that

(1) $x \notin H(t, x)$ for $t \in[0,1)$ and $x \in \operatorname{Bd} U$.

In fact, if $x \in H(t, x)=t G x=(t \lambda) F x$ for some $t \in(0,1)$, then $t \lambda \in(0,1)$ and $x \in A \subset U$. Since $U$ is open, we should have $x \notin \operatorname{Bd} U$. On the other hand, if $x \in H(0, x)=\{0\}$, then $x \notin \operatorname{Bd} U$.

(2) $H(0, x) \cap\{\lambda x: \lambda>1\}=\emptyset$ for $x \in \operatorname{Bd} U$.

In fact, $H(0, x)=\{0\}$. Since $x \in \operatorname{Bd} U$ and $U$ is open, we have $x \neq 0$ and $\lambda x \neq 0$ for $\lambda>1$.

Therefore, by Theorem 3, there is a $z \in \bar{U}$ such that $z \in H(1, z)=G z$. This completes our proof. 
Particular forms. 1. Schaefer [Sc1] first obtained Corollary 3.1 for a complete locally convex t.v.s. $E$ and a completely continuous map $\psi: E \rightarrow E$ (that is, $\psi$ is continuous and $\overline{\psi(n U)}$ is compact for each neighborhood $U$ of 0 and for each $n=1,2, \ldots)$.

2. For $F \in \mathbb{K}(E, E)$, Corollary 3.1 is due to Šeda [Se, Theorem 2].

From the proof of Corollary 3.1, we have the following:

Corollary 3.2. Let E, F, and $H$ be the same as in Corollary 3.1. Then either there exists a fixed point of $F$, or the set $\{x \in E: x \in \mu F x$ for some $\mu \in(0,1)\}$ is not bounded.

Particular form. Górniewicz, Granas, and Kryszewski [GGK, Corollaire 2] obtained Corollary 3.2 for a normed vector space $E$ and a compact map $F: E \rightarrow 2^{E}$ such that $\left.F\right|_{A} \in \mathbb{M}_{\mathrm{c}}(A, E)$ for each bounded subset $A$ of $E$.

\section{REFERENCES}

[B] F. E. Browder, Problèmes Non-Linéaires, Sém. Math. Supér., Presses Univ. Montréal, 1966.

[FP] P. M. Fitzpatrick And W. V. Petryshyn, Fixed point theorems and the fixed point index for multivalued mappings in cones, J. London Math. Soc. (2) 12 (1975), $75-85$.

[FM] M. Furi And M. Martelli, A degree for a class of acyclic-valued vector fields in Banach spaces, Ann. Scuola Norm. Sup. Pisa (4) 1 (1974), 301-310 (1975).

[GGK] L. Górniewicz, A. Granas et W. Kryszewski, Sur la méthode de l'homotopie dans la théorie des points fixes pour les applications multivoques (Partie 1: Transversalité topologique), C. R. Acad. Sci. Paris 307 (1988), 489-492.

[Gr1] A. Granas, Theorems on antipodes and theorems on fixed points for a certain class of multi-valued mappings in Banach spaces, Bull. Acad. Polon. Sci. 7 (1959), 271-275.

[Gr2] - Sur la méthode de continuité de Poincaré, C. R. Acad. Sci. Paris 282 (1976), 983-985.

[Gr3] _ On the Leray-Schauder alternative, Topol. Methods Nonlinear Anal. 2 (1993), 225-231.

[H] S. HAhn, Fixpunktsätze für mengenwertige Abbildungen in lokalkonvexen Räumen, Math. Nachr. 73 (1976), 269-283.

[KW] T. KaCZyŃski AND J. WU, A topological transversality theorem for multi-valued maps in locally convex spaces with applications to neutral equations, Canad. J. Math. 44 (1992), 1003-1013.

[Kn] S. KANIEL, Quasi-compact non-linear operators in Banach space and applications, Arch. Rational Mech. Anal. 20 (1965), 259-278.

[LS] J. Leray et J. Schauder, Topologie et équations fonctionnelles, Ann. Ecole Norm. Sup. (3) 51 (1934), 45-78.

[M] T.-W. MA, Topological degrees of set-valued compact fields in locally convex spaces, Dissertationes Math. 92 (1972).

[Mr] M. MARTELLi, Some results concerning multi-valued mappings defined in Banach spaces, Atti Accad. Naz. Lincei Rend. 54 (1973), 865-871. 
[N] M. Nagumo, Degree of a mapping in a convex linear space, Amer. J. Math. 73 (1951), 497-510.

[P1] S. PARK, Fixed point theory of multifunctions in topological vector spaces, II, J. Korean Math. Soc. 30 (1993), 413-431.

[P2] Foundations of the KKM theory via coincidences of composites of upper semi-continuous maps, J. Korean Math. Soc. 31 (1994), 493-519.

[PK] S. PARK AND H. KIM, Admissible classes of multifunctions on generalized convex spaces, Proc. Coll. Natur. Sci., Seoul Nat. Univ. 18 (1993), 1-21.

[Po] A. J. B. PotTer, An elementary version of the Leray-Schauder theorem, J. London Math. Soc. (2) 5 (1972), 414-416.

[Pw] M. J. Powers, Lefschetz fixed point theorems for multi-valued maps, Lecture Notes in Math., vol. 151, Springer-Verlag, Berlin, 1970, pp. 74-81.

[R] S. REICH, A remark on set-valued mappings that satisfy the Leray-Schauder conditions, Atti Accad. Naz. Lincei Rend. 61 (1976), 193-194; II, 66 (1979), 1-2.

[Sc1] H. Schaefer, Über die Methode der a priori Schranken, Math. Ann. 129 (1955), $415-416$.

[Sc2] _ Neue Existenzsätze in der Theorie nichtlinearer Integralgleichungen, Ber. Verhandl. Sächs. Akad. Wiss. Leipzig Math.-Natur. Kl. 101 (1955), no. 7, 40 pp.

[Se] V. ŠEdA, Some fixed point theorems for multivalued mappings, Czechoslovak Math. J. 39 (114) (1989), 147-164.

[Y] S. Yamamuro, Some fixed point theorems in locally convex linear spaces, Yokohama Math. J. 11 (1963), 5-12

Manuscript received July 15, 1994

SEHIE PARK

Department of Mathematics

Seoul National University

Seoul 151-742, KOREA

E-mail address: shpark@math.snu.ac.kr. 